\title{
Etnobotânica de Plantas Medicinais numa Escola Pública do Município de Capistrano, Ceará, Brasil
}

\author{
$\underline{\text { Arlindo Pereira Nogueiral }}^{l}$ \\ \arlindonoguevra@gmail.com
}

1. Universidade da Integração Internacional da Lusofonia Afro-Brasileira (UNILAB), Ceará - Brasil.

\author{
Histórico do Artigo:
}

Recebido em: 16 de julho de 2019 Aceito em: 16 de dezembro de 2019 Publicado em: 28 de dezembro de 2019

Resumo: Este estudo teve por objetivo realizar um levantamento das plantas medicinais cultivadas numa escola de ensino fundamental no município de Capistrano/CE e comparar as informações obtidas com as já descritas na bibliografia científica específica, visando o uso seguro em sua utilização. Trata-se de uma pesquisa- ação de abordagem mista, realizada através de entrevista semiestruturada com o núcleo gestor da escola envolvendo perguntas sobre identificação das plantas medicinais, partes utilizadas de cada planta para essa finalidade, modo de preparo e suas respectivas utilidades. 0s resultados mostraram que as plantas da família Lamiaceae são de grande utilidade para fins medicinais. No que tange a parte da planta, a folha teve grande influência, destacandose como a mais utilizada (85,7\%). Quando se refere ao método de preparo desses fitoterápicos, a decocção foi apontada em 100\% entre as indicações dos entrevistados. Constatou-se também, que 71,4\% de indicações terapêuticas citadas na escola estão de acordo com as recomendadas da Agência de Vigilância Sanitária- ANVISA. Assim, o conhecimento popular não pode ser rejeitado no que refere ao uso de plantas medicinais, entretanto, podendo ser utilizado como uma rica fonte de pesquisas científicas.

Palavras-chave: Conhecimento popular; Fitoterápicos; Educação; Capistrano- CE.

\section{Ethnobotany of Medicinal Plants in a Public School in the Municipality of Capistrano, Ceará, Brazil}

\begin{abstract}
This study aimed to conduct a survey of medicinal plants grown in an elementary school in the city of Capistrano / CE and compare the information obtained with those already described in the specific scientific literature, aiming at safe use in their use. This is a mixed approach action research, conducted through semistructured interviews with the school's management nucleus involving questions about identification of medicinal plants, parts used of each plant for this purpose, method of preparation and their respective utilities. The results showed that Lamiaceae plants are very useful for medicinal purposes. Regarding the plant part, the leaf had great influence, standing out as the most used (85.7\%). When referring to the method of preparation of these herbal medicines, the decoction was indicated in $100 \%$ among the indications of the interviewees. It was also found that $71.4 \%$ of therapeutic indications cited in the school are in accordance with the recommendations of the Health Surveillance Agency - ANVISA. Thus, popular knowledge cannot be rejected with regard to the use of medicinal plants, however, and can be used as a rich source of scientific research.
\end{abstract}

Keywords: Popular knowledge; Herbal medicines; Education; Capistrano- CE. 


\section{Etnobotánica de Plantas Medicinales en una Escuela Pública del Municipio de Capistrano, Ceará, Brasil}

Resumen: Este estudio tuvo como objetivo realizar una encuesta de plantas medicinales cultivadas en una escuela primaria en la ciudad de Capistrano / CE y comparar la información obtenida con las ya descritas en la literatura científica específica, con el objetivo de un uso seguro en su uso. Esta es una investigación de acción de enfoque mixto, realizada a través de entrevistas semiestructuradas con el núcleo de gestión de la escuela que involucra preguntas sobre la identificación de plantas medicinales, las partes utilizadas de cada planta para este propósito, el método de preparación y sus respectivas utilidades. Los resultados mostraron que las plantas de Lamiaceae son muy útiles para fines medicinales. En cuanto a la parte de la planta, la hoja tuvo una gran influencia, destacándose como la más utilizada (85.7\%). Al referirse al método de preparación de estos medicamentos a base de hierbas, la decocción se indicó en un 100\% entre las indicaciones de los entrevistados. También se encontró que el 71.4\% de las indicaciones terapéuticas citadas en la escuela están de acuerdo con las recomendaciones de la Agencia de Vigilancia Sanitaria - ANVISA. Por lo tanto, el conocimiento popular no puede ser rechazado con respecto al uso de plantas medicinales, sin embargo, y puede ser utilizado como una rica fuente de investigación científica.

Palabras clave: Conocimiento popular; Medicinas herbales; Educacion; Capistrano-CE.

\section{INTRODUÇÃO}

A utilização de espécies vegetais para fins medicinais remonta ao início da civilização, sendo que os primeiros registros referentes a plantas medicinais se encontram em textos chinês datados de 500 a.C. relatando nomes, doses e indicações de seu uso (KOVALSKI; OBARA, 2013; SANTOS-LIMA et al., 2016).

No Brasil seu uso foi estimulado por movimentos populares, diretrizes de várias conferências nacionais de saúde e por recomendações da Organização Mundial da Saúde- OMS (ANTONI0 et al., 2014). Dessa forma, em 2006 o Governo Federal brasileiro criou a Política Nacional de Plantas Medicinais e Fitoterapia, cujo objetivo é "garantir à população brasileira 0 acesso seguro e o uso racional de plantas medicinais e fitoterápicos, promovendo o uso sustentável da biodiversidade, o desenvolvimento da cadeia produtiva e da indústria nacional" (BRASIL, 2006, p. 20).

Desde então, uso de plantas para tratamento de doenças vem ganhando reconhecimento, recebendo seu devido valor pela ciência à medida que ela vai percebendo sua função fundamental para o desenvolvimento dos povos, oferecendo elementos práticos para outros pesquisadores das áreas de fitoquímica e farmacologia, favorecendo a descoberta de novos medicamentos (ALBUQUERQUE, 2005).

Adicionalmente, Hasenclever et al. (2017, p. 2) ponderam que a indústria de fitoterápicos se encontra em franco desenvolvimento a nível mundial, tornando-se, dessa forma, uma grande 
Etnobotânica de Plantas Medicinais numa Escola Pública do Município de Capistrano, Ceará, Brasil

oportunidade de tornar notório a riqueza natural e fatores em biodiversidade que temos no Brasil, visto que, contamos com muito conhecimento tradicional e científico acumulado sobre as atividades biológicas dessas plantas.

Contudo, essa temática ainda é escassa na literatura, principalmente quando comparamos à riqueza da biodiversidade que nosso país comporta. Pode-se citar o baixo interesse acadêmico, a desvalorização das plantas medicinais como tema de pesquisa e a falta da integração de pes $\neg$ quisadores de diferentes áreas de conhecimento, como algumas possíveis causas dessa escassez (CACCIA-BAVA et al., 2017).

Segundo Kovalski e Obara (2013), estima-se que o uso de plantas medicinais ainda seja o principal recurso para o tratamento de doenças em muitas comunidades, sendo muitas vezes, uma grande fonte de renda para essas famílias. No entanto, quando essa prática é conduzida de maneira indiscriminada, torna-se preocupante quando muitas pessoas as utilizam sem saber do risco, pois muitas destas plantas apresentam toxicidade, a exemplo da Anarcadium 0ccidentale (Cajú) e Dioscorea alata L. (Inhame) (FLORES, et al., 2001), Mangifera indica (Manga) (BOSCOL0; VALLE, 2008) e Ruta graveolens L. (Arruda) (VEIGA- JUNIOR et al., 2005), que precisam ser utilizadas de maneira correta, de preferência com acompanhamento médico

Ciente de tal realidade, este estudo propôs-se a realizar um levantamento das plantas medicinais cultivadas numa escola de ensino fundamental no município de Capistrano/CE, e comparar seu uso na referida escola com as já descritas na bibliografia científica específica. A intensão é que a partir desse estudo, sua aplicação esteja sendo feita de forma segura nesse espaço educacional.

\section{MATERIAL E MÉTODOS}

0 presente estudo trata-se de uma pesquisa-ação de abordagem mista. Este tipo de estudo constitui-se de uma importante ferramenta metodológica no contexto educacional, visto que se sustenta em abordagens que pressupõem a integração dialética entre pesquisador e pesquisado, entre teoria e ação (ROCHA, 2012). Foi realizado na Escola de Ensino Fundamental, Padre José de Anchieta, localizada na comunidade de Cajuás de Cima, aproximadamente 15 km do centro do município de Capistrano- $\mathrm{CE}$, com acesso pelas comunidades de Boqueirão, Onofre e Cajuás de Baixo. Este município está localizado a 120 km de Fortaleza, Capital do Estado, com área de 194,797 km² e aproximadamente 17.621 habitantes, possuindo uma maior população na 
zona rural tendo como principal fonte de renda, a agricultura, indústrias de produtos alimentares e comércio (IBGE, 2017).

A escola de Ensino Fundamental, Padre José de Anchieta fica aproximadamente a $15 \mathrm{~km}$ do centro do município de Capistrano comportando 7 salas, 01 auditório, 04 banheiros, 01 cantina e 02 almoxarifados, atendendo 216 alunos distribuídos entre Ensino Infantil, Fundamental e Ensino de Jovens e Adultos-EJA, através da prestação de serviços de 27 funcionários distribuídos entre núcleo gestor, professores e auxiliares de serviços gerais.

Na escola há uma horta que cultiva plantas medicinais que são utilizadas no preparo de chás que por sua vez são consumidos por funcionários e discentes. 0 manejo da horta envolve professores, alunos e funcionários, quando estes preparam o solo e utilizam recursos naturais, como o esterco bovino para adubação das plantas e água para regar a horta (Figura 1). Dessa forma, esse conhecimento tradicional é repassado para os discentes por meio da prática e explicado a forma de uso e finalidade de cada planta medicinal.
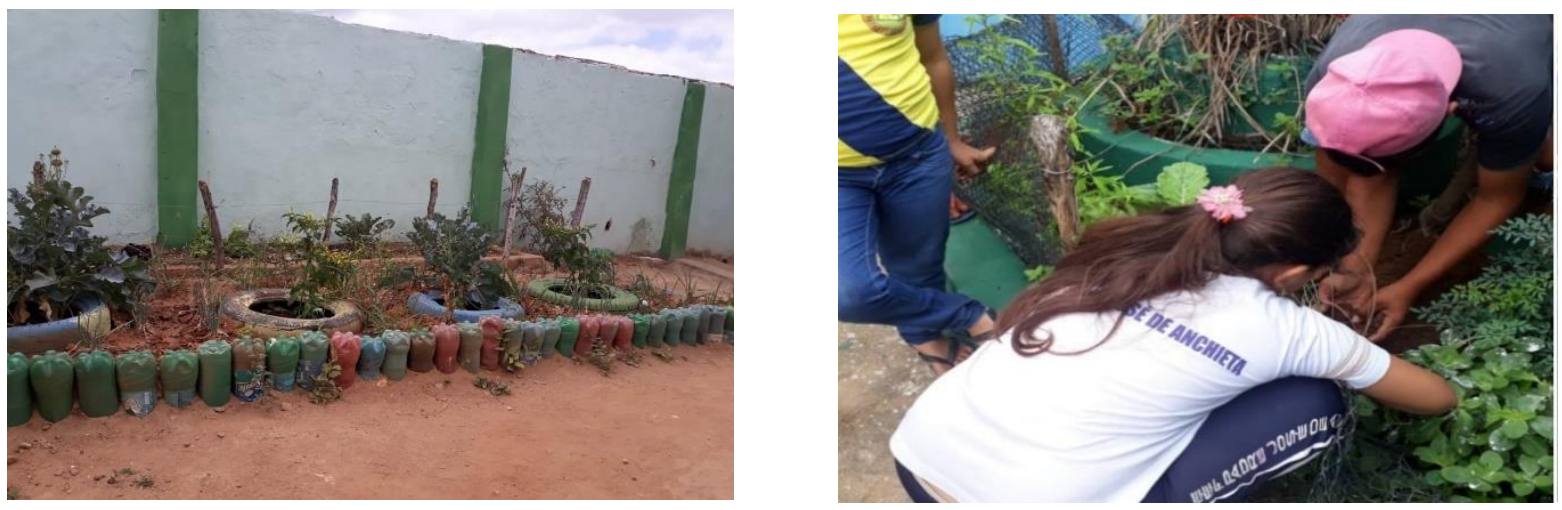

Figura 1: Plantação de mudas de plantas medicinais.

Fonte: acervo E.E.F Padre José de Anchieta, 2018.

Na fase 3, os artigos foram analisados individualmente aplicando os seguintes filtros: eliminando a duplicidade usando o gerenciador de referência (Mendeley); artigos cujo título não estava relacionado com a área; artigos pertencentes a capítulos de livro ou conferências devido à ausência do fator de impacto. Para finalizar essa etapa, as palavras-chave e os resumos foram analisados para verificar se o artigo estava ou não relacionado com a área. A coleta de dados ocorreu no mês de maio de 2018, por meio de entrevista, através de um questionário semiestruturado com perguntas abertas e fechadas e visita em lócus. Para isso, foi contatado ao núcleo gestor da escola, composto por diretor e dois coordenadores pedagógicos, e o professor de Ciências. A consulta ao professor de Ciências justifica-se por ser a disciplina que 
Etnobotânica de Plantas Medicinais numa Escola Pública do Município de Capistrano, Ceará, Brasil

trabalha com mais frequência a temática na escola, seja por aulas teóricas, seja por práticas na horta de plantas medicinais da escola.

Aos entrevistados foi explicitado os objetivos da pesquisa, garantindo-lhes o direito do anonimato das informações concedidas. Dessa forma, para a entrevista foi seguido um roteiro sobre: a) quais plantas medicinais são cultivadas na escola; b) nomes populares das plantas medicinais cultivadas na escola; c) Parte da planta utilizada para preparo do chá ou outra finalidade, e; d) modo de preparo. 0 uso do questionário tem sido muito utilizado pelos pesquisadores da atualidade, visto que, é um instrumento de pesquisa ágil e de baixo custo (PRODANOV; FREITAS, 2013).

Após a coleta das informações, foi feito o reconhecimento da nomenclatura botânica das plantas através de consulta bibliográfica especializada (SILVA; MARINE; MEL0, 2015; ANVISA, 2018; KOVALSKI; OBARA, 2013; OLIVEIRA; LUCENA, 2015). Posteriormente, as espécies foram ordenadas em unidades de análises e apresentadas em tabelas, conforme a mostram as Tabelas l e 2, cuja finalidade é discutir os resultados obtidos com os já descritos na literatura.

\section{RESULTADOS E DISCUSSÃ0}

0 levantamento evidenciou 07 espécies, distribuídas em 05 famílias botânicas (Tabela 1), as quais foram identificadas pelas famílias botânicas, nome científico e etnoespécie (conforme o conhecimento dos entrevistados) seguido de partes utilizadas de cada planta, forma de uso e suas indicações terapêuticas apontadas pelos entrevistados, baseado nos conhecimentos tradicionais e adquiridos entre gerações e que são aplicados na escola.

Verificou-se que o maior número de espécies encontradas pertence à família Lamiaceae $(42,7 \%)$. A grande representatividade dessa família também foi verificada em outros estudos etnobotânicos recentes (GOIS et al., 2016; FREITAS et al., 2015; SILVA et al., 2015; OLIVEIRA; LUCENA, 2015).

A justificativa da Lamiaceae ter uma grande representatividade dar-se devido ao poder de adaptação desta, tanto em ambientes tropicais quanto aos temperados, variabilidade de óleos essenciais que ela possui (OLIVEIRA; LUCENA, 2015) e seu uso medicinal (VIEIRA et al., 2015; NET0 et al., 2014).

No que tange as espécies registradas na escola, 71,4\% delas, (Tabela 2), são de uso recomendado, desde que, sejam utilizadas da maneira correta (ANVISA, 2018). 
Quanto a parte utilizada da planta, verificou-se a folha em 85,7\% entre as espécies registradas. Esse registro também foi encontrado em outros estudos que apontam a folha como a parte da planta mais utilizada para fins medicinais. Ribeiro et al. (2014) ao estudar o potencial terapêutico e uso de plantas medicinais em uma área de Caatinga no estado do Ceará, nordeste do Brasil, encontrou a folha como parte utilizada, correspondendo a 53,4\% das espécies estudadas. Já Gois, (2016) em seu estudo sobre a etnobotânica de espécies vegetais medicinais, encontrou a folha em 65\% entre as espécies estudadas. Da mesma forma, Oliveira e Lucena (2015) afirmam que das partes das plantas utilizadas pelos entrevistados de seu estudo, quanto ao uso de plantas medicinais por moradores de Quixadá-Ceará, as folhas representaram 89,4\% das citações pelos entrevistados.

Nesse sentido, Belizário e Silva (2012), dizem que o uso da folha é de fácil acesso e ainda promove a conservação do recurso vegetal, pois não impede o desenvolvimento e a reprodução da planta, caso a retirada da parte aérea não seja excessiva. Para Freitas et al. (2015), o uso significativo da folha entre as plantas medicinais deve-se a disponibilidade que elas têm durante 0 ano todo. Outro fator importante, que explica 0 uso da folha, como a mais consumida é que existem estudos que apontam a folha como a parte das espécies vegetais, onde tende a concentrar os princípios ativos, ou seja, o composto responsável pelo efeito farmacológico dessa planta (STEPP; MOERMAN, 2001).

No que se refere ao modo de preparo, 100\% das espécies é feito por decocção, (Tabela 1), processo no qual as partes da planta são fervidas junto com a água por alguns minutos. Resultados semelhantes foram encontrados por Freitas et al. (2015) ao estudar os quintais da comunidade de São João da Várzea em Mossoró, RN, por Ribeiro et al. (2014) quando pesquisou sobre o uso de plantas medicinais em uma área de Caatinga no estado do Ceará, e Neto et al. (2014) quando estudou a etnobotânica de plantas medicinais utilizadas pela Comunidade do Sisal no município de Catu, Bahia

No entanto, alguns estudos não apontam esse método de preparo como o mais adequado. Para Martins et al. (2000) deve-se ter cuidado com essa forma de preparo, pois essa prática pode resultar em efeitos inesperados devido as interações que ocorrem entre os constituintes químicos das plantas. Segundo as recomendações da Anvisa (2018), a decocção é indicada para partes de drogas vegetais com consistência rígida, tais como cascas, raízes, rizomas, caules, sementes e folhas coriáceas, indicando a infusão para partes de drogas vegetais de consistência menos rígida tais como folhas, flores, inflorescências e frutos, ou que contenham substâncias 
Etnobotânica de Plantas Medicinais numa Escola Pública do Município de Capistrano, Ceará, Brasil

ativas voláteis. A infusão consiste em verter água fervente sobre a droga vegetal e, em seguida, tampar ou abafar o recipiente por tempo determinado.

Tabela 1. Espécies, partes utilizadas e forma de preparo de plantas medicinais na E.E.F. Padre José de Anchieta, 2018.

\begin{tabular}{|c|c|c|c|}
\hline Família/Espécie & Etnoespécie & Parte utilizada & Preparo \\
\hline \multicolumn{4}{|l|}{ LAMIACEAE } \\
\hline Mentha arvensis L. & Menta & Caule, flor, folhas & Decocção \\
\hline Mentha X piperita L & Hortelã-menta & Caule, flor, folhas & Decocção, \\
\hline $\begin{array}{l}\text { Plectranthus barbatus } \\
\text { Andrews }\end{array}$ & Boldo & Folhas & Decocção \\
\hline \multicolumn{4}{|l|}{ POACEAE } \\
\hline Cymbopogon citratus (DC.) Stapf & Capim santo & Folhas & Decocção \\
\hline \multicolumn{4}{|l|}{ CRASSULACEAE } \\
\hline DKalanchoe brasiliensis Cambess. & Corama & Caule, folhas & Decocção \\
\hline \multicolumn{4}{|l|}{ VERBENACEAE } \\
\hline $\begin{array}{l}\text { Lippia alba (Mill.) N. E. Br. } \\
\text { ex Britton \& P. Wilson }\end{array}$ & Erva cidreira & Caule, flor, folhas & Decoç̧ão \\
\hline \multicolumn{4}{|l|}{ APIACEAE } \\
\hline Pimpinella anisum L. & Erva doce & Semente & Decocção \\
\hline Total & 07 & 04 & 01 \\
\hline
\end{tabular}

Fonte: Elaborado pelo autor, 2018.

Ao analisar as indicações terapêuticas, segundo as informações dos entrevistados, foram encontradas algumas espécies que não se encontram na lista de recomendações da Anvisa (2018), a saber, Kalanchoe brasiliensis Cambess (Corama) e Mentha arvensis L (Menta), conforme mostra a Tabela 2.

Oliveira et al. (2014) evidenciou em seu estudo sobre o uso de plantas medicinais com a terapêutica anticâncer, que é comum a maioria dos entrevistados compartilhar a opinião errônea de que plantas medicinais não fazem mal, sendo que a indicação terapêutica das plantas 
ocorre, principalmente, pela informação de familiares ou amigos, ignorando, muitas vezes, os profissionais da saúde neste processo.

Nesse sentido, Veiga Júnior et al. (2005) ponderam que do ponto de vista científico, muitas plantas utilizadas popularmente com finalidade medicinal possuem substâncias tóxicas, como óleos voláteis, alcaloides e saponinas (FLORES et al., 2001). Dessa forma, devem ser utilizadas com cuidado, considerando seus riscos toxicológicos.

Entretanto, ao se comparar as indicações terapêuticas da escola com as indicações da Anvisa (2018) (Tabela 2), constatou-se que diante das plantas medicinais citadas entre os entrevistados, $71,4 \%$ são de uso recomendados e não causam prejuízos à saúde.

Tabela 2. Indicações terapêuticas citadas na E.E.F. Padre José de Anchieta, e recomendações segundo a ANVISA, 2018.

\begin{tabular}{|c|c|c|}
\hline Família/Espécie & Etnoespécie & Indicação na escola \\
\hline \multicolumn{3}{|l|}{ LAMIACEAE } \\
\hline Mentha arvensis L. & Menta & Dor de cabeça; gripe. \\
\hline Mentha X piperita L & Hortelã-menta & Dor de barriga; febre. \\
\hline \multicolumn{3}{|l|}{ Plectranthus barbatus } \\
\hline Andrews & Boldo & Doenças \\
\hline intestinais. & Antidispéptico. & \\
\hline \multicolumn{3}{|l|}{ POACEAE } \\
\hline Cymbopogon citratus (DC.) Stapf & Capim santo & Gripe; calmante \\
\hline \multicolumn{3}{|l|}{ CRASSULACEAE } \\
\hline \multirow[t]{2}{*}{ Kalanchoe brasiliensis Cambess. } & Corama & Gripe. \\
\hline & $\begin{array}{l}\text { Não há } \\
\text { indicação }\end{array}$ & \\
\hline VERBENACEAE & & \\
\hline
\end{tabular}

Nesse sentido, Xavier e Flôr (2015) ponderam que os saberes populares é algo manifestado em determinado local ou grupo coletivo, através da prática cultural, sendo o consumo de chás medicinais um exemplo disso, visto que, esses saberes são conhecidos como um conjunto de conhecimentos elaborados a partir de experiências, crenças e superstições. Dessa forma, deve-se considerar que o conhecimento científico não é a única forma de educação devendo ser explorada novas possibilidades por meio do conhecimento popular.

Silva e Neto (2015) ponderam que não cabe nenhum tipo de impedimento ou tensão na relação entre o saber popular e o saber científico, sendo que ambos os tipos de saberes 
Etnobotânica de Plantas Medicinais numa Escola Pública do Município de Capistrano, Ceará, Brasil

anunciam ajuda mútua e complementariedade para o conhecimento, desde que reconheçam seus limites e suas contribuições.

\section{CONCLUSÃ0}

Assim, este estudo verificou que as plantas da família Lamiaceae são de grande utilidade popular para fins medicinais sendo muitas delas indicadas pela Agência de Vigilância Sanitária, proporcionando, dessa forma, uma maior segurança na sua utilização.

Percebeu-se, também a predominância das folhas como a parte mais utilizada da planta havendo similaridades em vários estudos e que esse método, além preservar a planta, é destacado como a parte onde está localizado o princípio ativo, o que corrobora a grande utilidade popular desse método.

No entanto, foi encontrado, tanto neste estudo, quanto em outros relacionados, a decocção como forma de preparo mais utilizada, sendo a infusão a mais indicada pelos órgãos de saúde especializado.

Por fim, percebeu-se que a maioria das indicações terapêuticas registradas no presente estudo, encontra-se nas recomendações da Anvisa (2018), o que nos permite dizer que o conhecimento popular não pode ser rejeitado quando se refere ao uso de plantas como medicamento, podendo ser utilizado como uma rica fonte para estudos científicos.

Portanto, através estudo, pretende-se fazer parcerias com as autarquias municipais de saúde e educação do munícipio, visando conscientizar a escola estudada e demais grupos sociais, quanto ao uso correto de plantas medicinais, segundo as recomendações de órgãos de saúde especializado.

\section{REFERÊNCIAS BIBLIOGRÁFICAS}

ALBUQUERQUE, U. P. Introdução a etnobotânica. 2. ed. Rio de Janeiro: Interciência, 2005.

ANTONIO, G.D. et al. Fitoterapia na atenção primária à saúde. Rev Saúde Pública 2014;48(3):541-553.

ANVISA. AGÊNCIA NACIONAL DE VIGILÂNCIA SANITÁRIA. Formulário de Fitoterápicos Farmacopeia Brasileira. Primeiro Suplemento. $1^{\text {a }}$ edição. Brasília. 2018.

BELIZÁRIO, T.L.; SILVA, L.A. Abordagem etnobotânica no tratamento de parasitoses em comércios de fitoterápicos e numa comunidade rural em Uberlândia-MG. Enciclopédia Biosfera, v.8, n.15, p.1730-1739, 2012.

BOSCOLO, 0.H.; VALLE, L.S. Plantas de uso medicinal em Quissamã, Rio de Janeiro, Brasil. IHERINGIA: Série Botânica, v.63, n.2, p.263-277, 2008 . 
CACCIA-BAVA, M.C.G.G. et al. Disponibilidade de medicamentos fitoterápicos e plantas medicinais nas unidades de atenção básica do Estado de São Paulo: resultados do Programa Nacional de Melhoria do Acesso e da Qualidade da Atenção Básica (PMAQ). Ciência \& Saúde Coletiva, 22(5):1651-1659, 2017.

FLORES J.S. et al. Plantas de la flora yucatanense que provocan alguna toxicidad en el humano. Revista Biomédica, v.12, n.2, p. 86-96, 2001.

FORMULÁRIO DE FITOTERÁPICO FARMACOPEIA BRASILEIRA. 1. ed. Brasília, 2011. 126p. Disponível em: < http://www.anvisa.gov.br/hotsite/farmacopeiabrasileira/conteudo/Formulario_de_Fitoterapicos_da_Farmacopeia _Brasileira.pdf >. Acesso 30 mai. 2018.

FREITAS, A.V.L. et al. Diversidade e usos de plantas medicinais nos quintais da comunidade de São João da Várzea em Mossoró, RN. Rev. Bras. Pl. Med., Campinas, v.17, n.4, supl. II, p.845-856, 2015.

GOIS, M.A.F. et al. Etnobotânica de espécies vegetais medicinais no tratamento de transtornos do sistema gastrointestinal. Rev. Bras. Pl. Med., Campinas, v.18, n.2, p.547-557, 2016.

HASENCLEVER, L. et al. A indústria de fitoterápicos brasileira: desafios e oportunidades. Ciência \& Saúde Coletiva, 22(8):2559-2569, 2017.

Instituto Brasileiro de Geografia e Estatística (IBGE). Cidades. 2017. Disponível em: 〈http://www.cidades.ibge.gov.br/v3/cidades/municipio/2302909>.

KOVALSKI, M.L; OBARA. A.T. 0 estudo da Etnobotânica das plantas medicinais na escola. Ciênc. Educ., Bauru, v. 19, n. 4, p. 911-927, 2013.

LUCA, V.D. et al. Utilização de plantas medicinais no entorno do Parque Estadual da Serra Furada, Santa Catarina, Brasil: uma abordagem etnobotânica. Revista Brasileira de Biociências, v. 12, n.2, p. 59-65, 2014.

MARTINS, E.R. et al. Plantas Medicinais. Viçosa, Editora UFV. Organização Mundial de Saúde. 2000.

Ministério da Saúde. Política nacional de plantas medicinais e fitoterápicos. Brasília, 2006.

NETO, F.R.G. et al. Estudo Etnobotânico de plantas medicinais utilizadas pela Comunidade do Sisal no município de Catu, Bahia, Brasil. Rev. Bras. Pl. Med., Campinas, v.16, n.4, p.856-865, 2014.

OLIVEIRA, D.M.S.; LUCENA, E.M.P. 0 uso de plantas medicinais por moradores de Quixadá-Ceará. Rev. Bras. Pl. Med., Campinas, v.17, n.3, p.407-412, 2015.

OLIVEIRA, L.A.R. et al. Levantamento sobre o uso de plantas medicinais com a terapêutica anticâncer por pacientes da Unidade Oncológica de Anápolis. Rev. Bras. Pl. Med., Campinas, v.16, n.l, p.32-40, 2014.

PRODANOV, C. C.; FREITAS, E. C. Metodologia do Trabalho Científico: Métodos e Técnicas da Pesquisa e do Trabalho Acadêmico. $2^{\text {a }}$ edição. 2013.

RIBEIR0, D.A. et al. Potencial terapêutico e uso de plantas medicinais em uma área de Caatinga no estado do Ceará, nordeste do Brasil. Rev. Bras. Pl. Med., Campinas, v.16, n.4, p.912-930, 2014.

ROCHA, T.L. Viabilidade da utilização da pesquisa-ação em situações de ensino-apendizagem. 2012. Disponível em: http://www.fucamp.edu.br/editora/index.php/cadernos/article/viewFile/218/194. Acesso em: 10 dez. 2019.

SANTOS-LIMA, T.M. et al. Plantas medicinais com ação antiparasitária: conhecimento tradicional na etnia Kantaruré, aldeia Baixa das Pedras, Bahia, Brasil. Rev. Bras. Pl. Med., Campinas, v.18, n.l, supl. I, p.240-247, 2016.

SILVA, M.D.P. et al. Levantamento de plantas medicinais cultivadas no município de Solânea, agreste paraibano: reconhecimento e valorização do saber tradicional. Rev. Bras. Pl. Med., Campinas, v.17, n.4, supl. II, p.881-890, 2015.

SILVA, S.F; NET0, J.F.M. Saber popular e saber científico. Revista Temas em Educação, João Pessoa, v.24, n. 2, p. 137154, jul.-dez. 2015. 
Etnobotânica de Plantas Medicinais numa Escola Pública do Município de Capistrano, Ceará, Brasil

STEPP, J.R.; MOERMAN, D.E. The importance of weeds in ethnopharmacology. Journal of Ethnopharmacology, v.75, p.19-23, 2001.

VEIGA JÚNIOR, V.F. et al. Plantas medicinais: cura segura? Química Nova, v.28, n.3, p.519- 28, 2005.

VIEIRA, L.S. et al. Plantas medicinais conhecidas por especialistas locais de uma comunidade rural maranhense. Rev. Bras. Pl. Med., Campinas, v.17, n.4, supl. III, p.1061-1068, 2015.

XAVIER, P. M. A; FLÔR, C. C. C. Saberes populares e educação científica:

Um olhar a partir da literatura na área de ensino de ciências. Revista Ensaio | Belo Horizonte | v.17 | n. 2 | p. 308328 | maio-ago | 2015. 